\title{
Anti-Programmed Cell Death Protein 1 (PD-1) Immunotherapy for Metastatic Hepatocellular Carcinoma After Liver Transplantation: A Report of Three Cases
}

\author{
Ouissam Al Jarroudi ${ }^{1}$, Ayhan Ulusakarya ${ }^{2}$, Wathek Almohamad ${ }^{2}$, Said Afqir ${ }^{1}$, Jean-Francois Morere ${ }^{2}$ \\ 1. Medical Oncology, University Hospital Mohammed VI, Oujda, MAR 2. Medical Oncology, Assistance Publique - \\ Hôpitaux de Paris (AP-HP) Paul Brousse Hospital, Paris, FRA
}

Corresponding author: Ouissam Al Jarroudi, aljarroudi.ouissam@gmail.com

\begin{abstract}
The treatment of recurrent hepatocellular carcinoma (HCC) after liver transplantation is difficult due to the lack of effective treatment options. The available evidence on the emerging immunotherapy in liver transplantation is based on anecdotal experiences and requires additional investigations. To determine the efficacy and safety of immunotherapy in liver transplant recipients, we report three cases of recurrent metastatic HCC after liver transplantation who were treated with nivolumab as off-label salvage therapy.
\end{abstract}

Categories: Gastroenterology, Oncology, Transplantation

Keywords: hepatocellular carcinoma, liver transplantation, pd-1 inhibitor

\section{Introduction}

Hepatocellular carcinoma (HCC) is ranked first among primary hepatic cancers, involving more than half a million patients per year [1]. Several early-phase clinical trials have investigated the value of immunotherapy in advanced HCC, as it represents the example of inflammation-induced cancers [2]. Liver transplantation is the treatment of choice for localized HCC in the absence of contraindications [3]. The recurrence rate after liver transplantation for HCC, despite the strict eligibility criteria, is high, varying between $6 \%$ to $18 \%$ [4]. The treatment of recurrent HCC after liver transplantation is a real challenge for the clinical practice, given the absence of any therapeutic standards [5].

The principle of immunotherapy consists of the induction of an anti-tumor immune response using monoclonal antibodies targeting immune checkpoints present on the surface of T-cells. Immune-checkpoint inhibitors include two major families of antibodies targeting either the cytotoxic T-lymphocyte-associated antigen 4 (CTLA-4) or the programmed cell death protein 1 (PD-1) [6]. Nivolumab is the first recombinant human monoclonal antibody developed in the world that targets PD-1 [7]. The response rate with this molecule reached $20 \%$ with two complete responses in phase I/II trial (CheckMate-040 trial) in advanced HCC. These results seemed extremely promising for the advanced stage of this malignancy known by its poor survival [8]. Nivolumab is also characterized by its persistent effect in responders, which encouraged scientists-clinicians to continue patients' enrollment for this setting. At the Congress of the American Society of Clinical Oncology (ASCO) in 2017, the updated data of this study confirmed satisfactory results for overall survival (OS) with a median of 28.6 months in the first line of treatment and 15.6 months in the second line [9]. The nivolumab positioning in the first-line versus sorafenib is under investigation in phase III [10].

It is actually known that the tolerance of transplant organs involves the modulation of cell-mediated immunity [11-12] and that the transplant rejection's risk may be caused by the deregulation of these processes [13]. The fear of transplant rejection leads to the exclusion of the recipient's solid organ transplant from the clinical trials of immune checkpoints inhibitors [14]. The current data on immunotherapy after hepatic transplantation are lacking and are limited to case reports and small published series [15-16].

In this paper, we describe three cases of recurrent metastatic HCC after liver transplantation in whom nivolumab was used as off-label salvage treatment.

\section{Case Presentation}

\section{Case reports}

Case 1

A 70-year-old male with a personal history of nonalcoholic steatohepatitis presented with multifocal HCC that was diagnosed at the age of 64 . No regional lymph node or distal metastasis was found, classified as 
Child A and model for end-stage liver disease (MELD) 7. He was treated with three courses of arterial chemoembolization, followed by a complete hepatectomy and an orthotopic liver transplant. After 33 months of transplantation, the patient had rising alpha-fetoprotein and the presence of liver metastasis on surveillance imaging. The diagnosis consisted of recurrent metastatic HCC. The patient received sorafenib as first-line treatment. As a second-line treatment, the patient received six cycles of gemcitabine and oxaliplatin. However, his disease progressed according to Response Evaluation Criteria in Solid Tumors (RECIST), and he was treated with regorafenib. After metastatic disease progression, the patient was treated by salvage nivolumab at $240 \mathrm{mg}$ every two weeks. The patient continued the immunosuppression of tacrolimus. The patient presented right-sided abdominal pain, and liver enzymes and total bilirubin were elevated after four doses of nivolumab. The patient was then treated with a pulse of high-dose steroids. Therefore, nivolumab treatment was interrupted. The patient was followed up and died four months after tumor progression.

Case 2

A 62-year-old female with a history of hepatitis B virus and multifocal HCC Child B and MELD 14, underwent liver transplantation. One year after, surveillance imaging revealed adrenal and pulmonary metastasis and mediastinal lymphadenopathy. She received first-line treatment using sorafenib for six months. Because of tumor progression, a second-line treatment by regorafenib was decided. However, the disease progressed and alpha-fetoprotein rose after nine months of second-line tyrosine kinase inhibitor (TKI). She was treated by chemotherapy (5 fluorouracil and oxaliplatin) for six cycles. After progression, a multidisciplinary teambased discussion decided to start nivolumab while maintaining immunosuppression by tacrolimus. She received five cycles of nivolumab $240 \mathrm{mg}$ every two weeks, with continuous monitoring of serum tacrolimus concentration and liver function. No graft rejection or immune adverse events was observed in this case. However, her metastatic disease progressed. A fifth-line treatment by lenvatinib was started. This patient is still alive with clinical-radiological disease stability.

Case 3

This was a 66-year-old male with a history of cirrhosis and hepatitis B virus, with Child B and MELD 20 HCC. After two courses of arterial chemoembolization, a total hepatectomy and orthotopic liver transplant were performed. Two years later, the patient presented with hepatic and pulmonary metastases. The patient was treated with first-line sorafenib for two years. A second-line treatment by Regorafenib was administrated for 9 months. After progression on chemotherapy (gemcitabine and oxaliplatin) used in the third-line, the patient received nivolumab every two weeks. He continued immunosuppression using tacrolimus. The PD-1 inhibition by nivolumab did not induce immune adverse events. His tumor progressed after six cycles of nivolumab and a new therapeutic line using cabozantinib has been started.

\section{Discussion}

Nivolumab was the first PD-1 inhibitor approved for HCC treatment by the Food and Drug Administration (FDA) in September 2017 because of the trial CheckMate 040 outcomes [2-8]. The nivolumab EMA approval has not yet been received even after CheckMate 459 trial data demonstrated clinical improvement in OS and overall response rate (ORR) with nivolumab compared to sorafenib in the first-line setting for advanced HCC [10]. Initially, solid organ transplantations were the exclusion criteria in immune checkpoint inhibitors due to immunosuppressive agents administration [17-18].

The administration of nivolumab after liver transplantation poses the question of its tolerance and efficacity. In our three cases, no severe adverse effects with nivolumab were observed. Notably, liver transplant recipients may, therefore, be included in immunotherapy clinical trials. However, appropriate patient selection, strict laboratory, clinical monitoring, and the use of low immunosuppressive drug dosing during nivolumab therapy are needed.

Immunosuppression and immunotherapy are two crucial conditions in the identification of patients that may receive immunomodulatory agents in this setting [19]. However, the immunogenicity of transplant organs differs. It appears that the liver grafts are less immunogenic as compared to other organs, such as the heart, the kidneys, or the lungs, which leads us to discuss the possibility of less aggressive immunosuppressive treatment use [20].

None of our patients showed a significant benefit under immunotherapy, probably due to a late introduction in the third or fourth-line for aggressive diseases. The small patient sample does not support our conclusion on the efficacy of immunotherapy in hepatic transplant patients. Further studies with a large patient cohort are awaited.

\section{Conclusions}

Overall, the use of immunotherapy in HCC recurrent after liver transplantation is not recommended in light of current data but medicine is one of the most evolving areas where the guidelines change overnight.

Therefore, to define the feasibility of immunotherapy in hepatic transplants for HCC, clinical trials including 
this specific population are needed.

\section{Additional Information Disclosures}

Human subjects: Consent was obtained by all participants in this study. Conflicts of interest: In compliance with the ICMJE uniform disclosure form, all authors declare the following: Payment/services info: All authors have declared that no financial support was received from any organization for the submitted work. Financial relationships: All authors have declared that they have no financial relationships at present or within the previous three years with any organizations that might have an interest in the submitted work. Other relationships: All authors have declared that there are no other relationships or activities that could appear to have influenced the submitted work.

\section{Acknowledgements}

The authors would like to thank Dr Vincent Castagne for his help in obtaining data on immunotherapy in HCC from hospital pharmacy registries. We also thank Dr Hamed Chaabouni and Dr Yusuf Gumus for their help in data collection and Dr Khalid El Bairi for manuscript revision.

\section{References}

1. El Serag HB: Hepatocellular carcinoma. N Engl J Med. 2011, 365:1118-1127. 10.1053/j.gastro.2007.04.061

2. Finkelmeier F, Czauderna C, Perkhofer L, et al.: Feasibility and safety of nivolumab in advanced hepatocellular carcinoma: real-life experience from three German centers. J Cancer Res Clin Oncol. 2019, 145:253-259.

3. Welker MW, Bechstein WO, Zeuzem S, Trojan J: Reccurent hepatocellular carcinoma after liver transplantation - an emerging clinical challenge. Transpl Int. 2013, 26:109-118. 10.1111/j.14322277.2012.01562.x

4. Yoo HY, Patt CH, Geschwind JF, Trojan PJ: The outcome of liver transplantation in patients with hepatocellular carcinoma in the United States between 1988 and 2001: 5-year survival has improved significantly with time. J Clin Oncol. 2003, 21:4329-4335. 10.1200/jco.2003.11.137

5. Au KP, Chok KSH: Multidisciplinary approach for post-liver transplant recurrence of hepatocellular carcinoma: a proposed management algorithm. World J Gastroenterol. 2018, 24:5081-5094. 10.3748\%2Fwjg.v24.i45.5081

6. Paradoll DM: The blockade of immune checkpoints in cancer immunotherapy . Nat Rev Cancer. 2012, $12: 252-264$.

7. Kudo M: Systemic therapy for hepatocellular carcinoma: latest advances . Cancers. 2018, 10:412 10.3390/cancers10110412

8. El-Khoueiry AB, Sangro B, Yau T, et al.: Nivolumab in patients with advanced hepatocellular carcinoma (CheckMate 040): an open-label, non-comparative, phase 1/2 dose escalation and expansion trial. Lancet. 2017, 389:2492-2502. 10.1016/S0140-6736(17)31046-2

9. Crocenzi TS, El-Khoueiry AB, Yau TC, et al.: Nivolumab (nivo) in sorafenib (sor)-naïve and -experienced pts with advanced hepatocellular carcinoma (HCC): CheckMate 040 study. J Clin Oncol. 2017, 35:4013-4013.

10. Sangro B, Park JW, Dela Cruz CM, et al.: A randomized, multicenter, phase 3 study of nivolumab vs sorafenib as first-line treatment in patients (pts) with advanced hepatocellular carcinoma (HCC): CheckMate-459. J Clin Oncol. 2016, 34:4147.

11. Riella LV, Paterson AM, Sharpe AH, Chandraker A: Role of the PD-1 pathway in the immune response . Am J Transplant. 2012, 12:2575-2587. 10.1111/j.1600-6143.2012.04224.X

12. Tanaka K, Albin MJ, Yuan X, et al.: PDL1 is required for peripheral transplantation tolerance and protection from chronic allograft rejection. J Immunol. 2007, 179:5204-5210. 10.4049/jimmunol.179.8.5204

13. Zhang T, Fresnay S, Welty E, et al.: Selective CD28 blockade attenuates acute and chronic rejection of murine cardiac allografts in a CTLA-4-dependent manner. Am J Transplant. 2011, 11:1599-1609. 10.1111/j.1600-6143.2011.03624.x

14. Hodi FS, O'Day SJ, McDermott DF, et al.: Improved survival with ipilimumab in patients with metastatic melanoma. N Engl J Med. 2010, 363:711-723. 10.1056/NEJMoa1003466

15. Robert C, Thomas L, Bondarenko I, et al.: Ipilimumab plus dacarbazine for previously untreated metastaic melanoma. N Engl J Med. 2011, 364:2517-2526. DOI: 10.1056/NEJMoa1104621

16. De Toni EN, Gerbes AL: Tapering of immunosuppression and sustained treatment with nivolumab in a liver transplant recipient. Gastroenterology. 2017, 152:1631-1633. 10.1053/j.gastro.2017.01.063

17. Deleon TT, Salomao MA, Aqel BA, et al.: Pilot evaluation of PD-1 inhibition in metastatic cancer patients with a history of liver transplantation: the Mayo clinic experience. J Gastrointest Oncol. 2018, 9:1054-1062. 10.21037\%2Fjgo.2018.07.05

18. Liu M, Guo W, Zhang S: Cancer immunotherapy in patients with new or recurrent malignancies after liver transplantation. Int J Surg Oncol. 2017, 2:e45. 10.1097\%2FIJ9.0000000000000049

19. Rammohan A, Reddy MS, Farouk M, Vargese J, Rela M: Pembrolizumab for metastatic hepatocellular carcinoma following live donor liver transplantation: the silver bullet?. Hepatology. 2018, 67:1166-1168.

20. Sanchez-Fueyo A, Strom TB: immunologic basis of graft rejection and tolerance following transplantation of liver or other solid organs. Gastroenterology. 2011, 140:51-64. 10.1053/j.gastro.2010.10.059 